(C) [2006] IEEE. Reprinted, with permission, from [Wenjing Jia, Huaifeng Zhang, Xiangjian He, and Qiang Wu, A Comparison on Histogram Based Image Matching Methods, Video and Signal Based Surveillance, 2006. AVSS '06. IEEE International Conference on, Nov. 2006]. This material is posted here with permission of the IEEE. Such permission of the IEEE does not in any way imply IEEE endorsement of any of the University of Technology, Sydney's products or services. Internal or personal use of this material is permitted. However, permission to reprint/republish this material for advertising or promotional purposes or for creating new collective works for resale or redistribution must be obtained from the IEEE by writing to pubs-permissions@ieee.org. By choosing to view this document, you agree to all provisions of the copyright laws protecting it 


\title{
A Comparison on Histogram Based Image Matching Methods
}

\author{
Wenjing Jia, Huaifeng Zhang, Xiangjian He, and Qiang Wu \\ Computer Vision Research Group, University of Technology, Sydney \\ PO Box 123, Broadway, NSW, 2007, Australia \\ \{wejia, hfzhang, sean,wuq\}@it.uts.edu.au
}

\begin{abstract}
Using colour histogram as a stable representation over change in view has been widely used for object recognition. In this paper, three newly proposed histogram-based methods are compared with other three popular methods, including conventional histogram intersection (HI) method, Wong and Cheung's merged palette histogram matching (MPHM) method, and Gevers' colour ratio gradient (CRG) method. These methods are tested on vehicle number plate images for number plate classification. Experimental results disclose that, the CRG method is the best choice in terms of speed, and the GWHI method can give the best classification results. Overall, the CECH method produces the best performance when both speed and classification performance are concerned.
\end{abstract}

\section{Introduction}

Using colour histogram as a stable representation over change in view has been widely used for object recognition. It was explored by Swain and Ballard [8][9], who introduced the colour indexing technique to efficiently recognise objects by matching their colour histograms via histogram intersection (HI) algorithm. This algorithm did not address the issue of illumination variation. Towards an illumination-insensitive histogram-based image matching algorithm, many alternations have been suggested. These methods could be roughly divided into two groups.

The first group tries to improve the way of measuring similarity of histograms in order to make the histogrambased matching algorithms more robust to illumination changes. Wong and Cheung [10] proposed a mergedpalette histogram matching (MPHM) method. Using their method, two perceptually similar colours, instead of identical colours, are able to be intersected, which has improved the robustness of the conventional $\mathrm{HI}$ algorithm to colour variations. In [6], Jia et al. proposed a Gaussian-weighted histogram intersection (GWHI) method, which applied a
Gaussian weight function to differentiate the contributions to the final matching from different colours. Since this algorithm needs a couple of seconds to match two images of $120 \times 40$. Later, a refined GWHI is proposed in [5].

The second group aims to generate histograms from other features instead of from colour values directly. These techniques include Funt and Finlayson's [2] ratio of colour RGB triples, and Nayar and Bolle's [7] colour reflection ratios. Gevers [3] further developed the colour ratio gradient (CRG) to make it insensitive to geometry and position of objects, shadow, illumination, and other imaging conditions. At the same time, these methods have introduced a slight amount of spatial information into their histogram representation. However, since this spatial information is defined on absolute position relationship, their performance on matching images of compound colour objects, such as vehicle number plates, is unsatisfactory. To address this problem, a novel colour edge cooccurrence histogram $(\mathrm{CECH})$ method is proposed in [4]. In this method, the histogram only counts the number of pairs of pixels which are located on two sides of edge points along their gradient direction lines and at a distance away from the edge points. This design not only employs the spatial information, but also keeps the flexibility of the histogram description.

Above methods all have claimed their advantages in their publications. In this paper, these methods are brought together and their performance in terms of speed and matching rates are compared on number plate images.

The remaining parts of this paper are organised as follows. In Section 2, the six methods to be compared in this paper are briefly introduced. In Secion 3, experiments are carried out on vehicle number plate images, and performance of each method is analysed and compared in terms of speed and matching rate. This paper concludes in Section 4.

\section{Histogram Based Image Matching Methods}

In this section, thee popular histogram-based image matching methods and other three newly developed methods are briefly introduced. 


\subsection{Conventional Histogram Intersection (HI) Method}

Histogram-based image matching algorithms try to measure the similarity in contents via their histograms between a model image and any images in database, i.e., target images, in order to properly classify or retrieve images. Histogram intersection (HI), proposed by Swain and Ballard [8][9], is a straightforward method to calculate the matching rate between two histograms for this purpose.

Assume the histograms of a model image and a target image are $H_{M}$ and $H_{T}$ respectively, and each contains $n$ bins. Swain and Ballard [9] defined the intersection $H I$ of two histograms as:

$$
H I=\sum_{i=1}^{n} \min \left(h_{M}(i), h_{T}(i)\right),
$$

where the subscripts " $\mathrm{M}$ " and "T" denote for "model" and "target" respectively, and both $H_{M}$ and $H_{T}$ are normalised, i.e., $\sum_{i=1}^{n} h_{M}(i)=1$ and $\sum_{i=1}^{n} h_{T}(i)=1$.

The resultant fractional matching value between 0 and 1 is actually the proportion of pixels from the model image that have corresponding pixels of the same colour in the target image. A higher histogram matching rate indicates higher similarity between the model image and the target image.

\subsection{Merged Palette Histogram Matching (MPHM) Method}

The conventional HI algorithm provides a straightforward and robust method to measure the similarity between two images. By such a way, the image matching problem is converted to a simple problem that, to what extent the histogram of the model image is similar to that of the target image.

However, this algorithm has a limitation due to the fact that it assumes identical colour matching, i.e., only corresponding bins of identical colours can be matched. In practice, however, colours of images can be distorted both in the scene itself and in the image capturing process. Hence, images with same visual information but with variant colour intensities may degrade the similarity level significantly.

In order to overcome this problem, Wong and Cheung [10] proposed a merged palette histogram matching (MPHM) method. The essence of their method is to extend the intersection from bins of identical colours to bins of similar colours. As long as the distance between two colours is less than a given threshold, the intersection between the bins of the two colours can be calculated. In this method, the histograms to be compared are not the original colour histograms but the ones defined on a common palette of two images. Perceptually similar colours in two images are firstly merged to generate the common palette. The conventional histogram intersection are then performed on a merged common palette. Using the MPHM method, two perceptually similar colours, instead of identical colours, are able to be intersected, which improves the robustness of the conventional $\mathrm{HI}$ algorithm to colour variations.

\subsection{Gaussian Weighted Histogram Inter- section (GWHI) Method}

Wong and Cheung's MPHM method has produced more robust image retrieval results for images captured under various illumination conditions [10]. But it assumes an identical weight of contributions from colours which have different similarities with the given colour. This, however, does not well reflect the matching contributed by different colours. In [6], a Gaussian weight function is utilised to differentiate the contributions from colours which have different distance to the given colour.

In the GWHI method, a truncated Gaussian weight function of colour distance is applied to the conventional HI method in order to describe the relationship between the colour distance and the weight as [6]:

$$
\begin{aligned}
& G W H I= \\
& \sum_{\vec{c}_{i} \in \mathbf{C}_{M}} \sum_{\vec{c}_{j} \in \mathbf{C}_{T}} \min \left(h_{M}\left(\vec{c}_{i}\right), h_{T}\left(\vec{c}_{j}\right)\right) \cdot \exp \left(-\frac{\left\|\vec{c}_{i}-\vec{c}_{j}\right\|^{2}}{2 \sigma^{2}}\right),
\end{aligned}
$$

where $-3.3 \sigma \leq\left\|\vec{c}_{i}-\vec{c}_{j}\right\| \leq 3.3 \sigma$.

The weight functions corresponding to the three methods are shown in Figure 1.

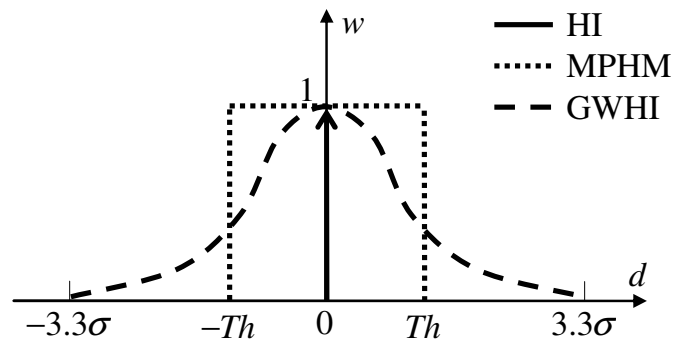

Figure 1. Weight functions in three histogram intersection methods.

The colour distance is defined as perceptual difference between two colours as:

$$
\left\|\vec{c}_{1}-\vec{c}_{2}\right\|=\sqrt{\left(l_{1}-l_{2}\right)^{2}+\left(u_{1}-u_{2}\right)^{2}+\left(v_{1}-v_{2}\right)^{2}}
$$

where $\vec{c}_{1}=\left(l_{1}, u_{1}, v_{1}\right)$ and $\vec{c}_{2}=\left(l_{2}, u_{2}, v_{2}\right)$ are two colours represented in CIE Luv colour space. 


\subsection{Refined GWHI Method}

The GWHI method has demonstrated better classification results in [6]. However, it usually needs a couple seconds to match two images with size of $120 \times 40$, which is obviously not acceptable for practical use. In order to improve its speed, a refined GWHI method is proposed in [5], where colour quantisation is first performed on both model and target images in order to reduce the dimension of the resultant colour histograms.

\subsection{Colour Ratio Gradients (CRG)}

The techniques introduced above all belong to the first group which aims to address the illumination-sensitive problem of the histogram-based image matching algorithms. Either the conventional HI method [8][9], the MPHM method [10], or the newly proposed GWHI method [6] and its refined version [5], does not include any spatial information in their histogram representations. Histograms in these approaches record only the number of pixels with certain colours. Therefore, they are easy to compute and insensitive to small changes in viewing positions. However, they are very likely to produce false positives because two apparently different images may have same colour histograms.

Another group of solutions aim to redefine the histogram representation using other features rather than using colour values directly. Funt and Finlayson [2] produced a new measurement based on the ratio of colour RGB triples in neighbouring area to locate objects. Other improved methods include illumination-independent colour reflection ratios proposed by Nayar and Bolle [7]. Gevers [3] further developed the colour ratio gradient (CRG) to make it insensitive to geometry and position of objects, shadows, illuminations, and other imaging conditions.

The three-channel colour ratio gradients on RGB colour space is defined in a small neighbourhood as [3]:

$$
\left\{\begin{aligned}
\nabla M_{R G} & =\sqrt{\left(\frac{R_{1} G_{2}-R_{2} G_{1}}{R_{2} G_{1}+R_{1} G_{2}}\right)^{2}+\left(\frac{R_{3} G_{4}-R_{4} G_{3}}{R_{4} G_{3}+R_{3} G_{4}}\right)^{2}} \\
\nabla M_{R B} & =\sqrt{\left(\frac{R_{1} B_{2}-R_{2} B_{1}}{R_{2} B_{1}+R_{1} B_{2}}\right)^{2}+\left(\frac{R_{3} B_{4}-R_{4} B_{3}}{R_{4} B_{3}+R_{3} B_{4}}\right)^{2}} \\
\nabla M_{G B} & =\sqrt{\left(\frac{G_{1} B_{2}-G_{2} B_{1}}{G_{2} B_{1}+G_{1} B_{2}}\right)^{2}+\left(\frac{G_{3} B_{4}-G_{4} B_{3}}{G_{4} B_{3}+G_{3} B_{4}}\right)^{2}}
\end{aligned}\right.
$$

In this formula, for simplification purpose, the colour values at four locations $p_{i}(i=1,2,3,4)$ are denoted as $R_{i}$, $G_{i}$, and $B_{i}$ respectively. The locations of $p_{i}$ in the small neighbourhood which is centred at point $p$ are illustrated in Figure 2.

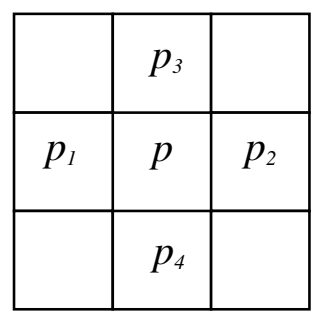

Figure 2. Locations of four neighbours that are involved in the computation of the colour ratio gradient at the central pixel $\vec{p}$.

\subsection{Colour Edge Cooccurrence Histogram (CECH) Method}

Gevers' CRG method introduces a slight amount of spatial information into the histogram representation and extended the adaptability of histogram-based image matching methods. However, this method applied an absolute position relationship, which is sensitive to the deformation of objects. Moreover, since the CRG is defined in a very compact area (see Figure 2), when applied to locate objects with relatively uniform textural surface, such as vehicle number plates, the method cannot effectively differentiate matching of images with different colours.

In [4], a novel colour edge cooccurrence histogram algorithm is proposed. This method only cares about the spatial-colour distributions near the edge points in order to improve the computation efficiency and also prevent solid colour regions from dominating the histogram. Moreover, in this method, the colour pairs are counted only for pairs of pixels which are located on two sides of edge points along their gradient direction lines, in order to make the histogram invariant to rotation and deformation of objects in images. Instead of employing the conventional histogram intersection to measure the similarities between CECH histograms, the GWHI is extended to take into account both the matching between identical colour pairs and the matching between similar colour pairs. In order to improve its processing speed, colour number reducing is performed at first, where Wu's [11] variance minimisation algorithm is (applied because of its high speed and nearly no perceptual colour distortion [4].

\section{Experiments}

In experiments, the above introduced six methods are tested on vehicle number plate images for number plate classification. The aim is to find a robust classification between different classes of number plate images. Two number plate images are viewed to belong to the same class 
when they have similar foreground and background colours, but they may have quite different content (characters), size and viewing condition. Two classes of number plates are frequently used in NSW, Australia. Without loss of generality, these two classes of vehicle number plate images are tested, namely, plates with yellow background and plates with white background.

\subsection{Data Sets}

A yellow number plate image is manually selected as the model image for yellow class, which is shown in Figures 3. The selection criterion is that the intensity of model images should not be too strong or too weak compared with other target images. Then, the above six image matching methods are used to compute matching with total 130 various number plate images, including 87 yellow plates and 43 white plates. All number plate images in experiments are with dimension of $120 \times 40$. Some examples of yellow and white target images are shown in Figures 4. It can be seen that, due to colour variations, images that belong to same class may have quite different appearances.

Moreover, for the purpose of comparison, the same $T h=15$ (see Figure 1) is set in the MPHM method, the GWHI method, its refined version, and the CECH method. In the CRG method, the colour ratio gradient in Equation 4, which takes value in the range of $[0, \sqrt{2}]$, is quantised to $[0,15]$.

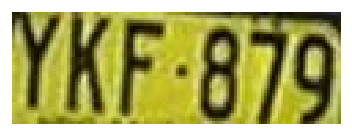
Figure 3. The yellow model image used in ex-
periments.

\subsection{Experimental Results and Discussion}

The matching results obtained using the six methods are plotted in Figure 5. The average time used for computing matching using the six methods are given in Table 1. All experiments are performed on a PC with Intel Pentium IV 1.8GHz CPU and 380MB of RAM.

As seen in Figure 5(a) that, using the conventional HI method, it is almost impossible to separate two classes of number plate images via their colour histograms. Using the MPHM method, this has been improved a lot. As shown in Figure 5(b), the matching rates between same class of number plates (inter-class) have all been increased, while those between different classes of number plates (inter-class) are still very low. However, as seen from the figure, the matching rates of same class of plates still spread in a wide range.

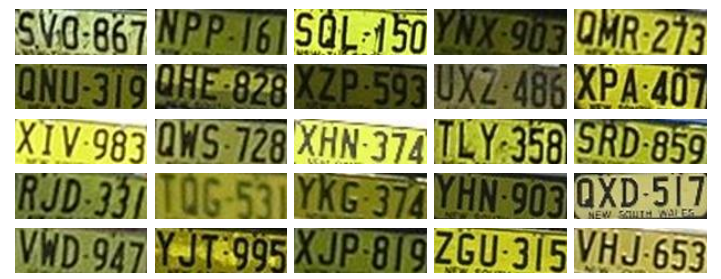

ASL.82R API.31N AKT.83日 ALK.86P AIU.06S
$\overline{\text { BMH.767 AJR.380 AGG.13Y SUP.338 AL } 0.79 \mathrm{Y}}$
ASI.74G AOH.17N ANG.11C AOT.67H AHE.63U

Figure 4. Examples of yellow target images (upper five rows) and white target images (lower three rows) used in experiments.

\section{Table 1. Time used for computing image matching using the six methods.}

\begin{tabular}{|c|c|}
\hline Methods & Time (in milliseconds) \\
\hline HI & 935 \\
\hline MPHM & 1085 \\
\hline GWHI & 1606 \\
\hline RGWHI & 12 \\
\hline CRG & 4 \\
\hline CECH & 22 \\
\hline
\end{tabular}

This shows the MPHM method is still very sensitive to colour variations.

Using the GWHI method, as shown in Figure 5(c), it is more easy to separate two classes of number plates, since the inter-class distance of the matching rates becomes much larger and the intra-class distance becomes much smaller than pervious two methods. The matching rates of number plates from same class are much more stable compared with the results obtained using the HI and MPHM methods. This is because a weight function is employed rather than simply adding up all intersections from different colours.

It is noted that from Table 1 that, computing matching using the three methods needs 1 to 2 seconds in average.

Using the refined GWHI method, the computation speed has been improved greatly. As shown in Table 1, the average time needed for matching two number plate images using the refined GWHI method is 12 milliseconds. Compared with the average time of 935 milliseconds in the HI method, 1085 milliseconds in the MPHM method, and 1606 milliseconds in the GWHI method, this processing speed is much faster. However, this higher speed is achieved at 


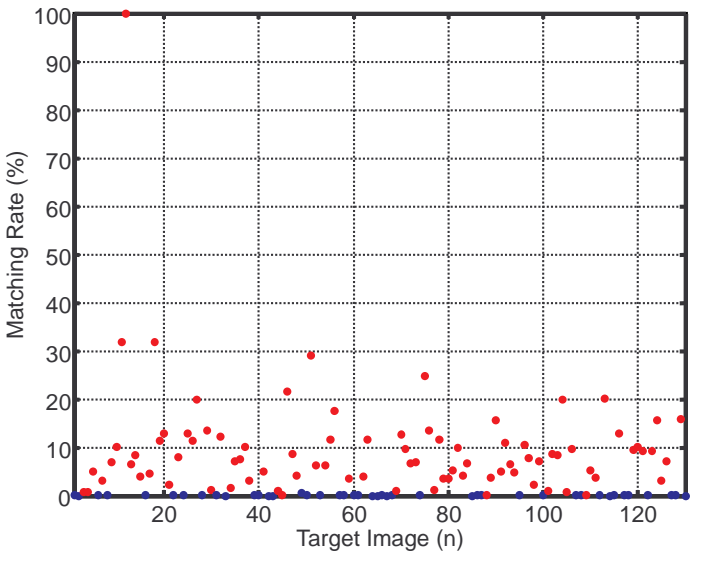

(a) using the conventional HI method

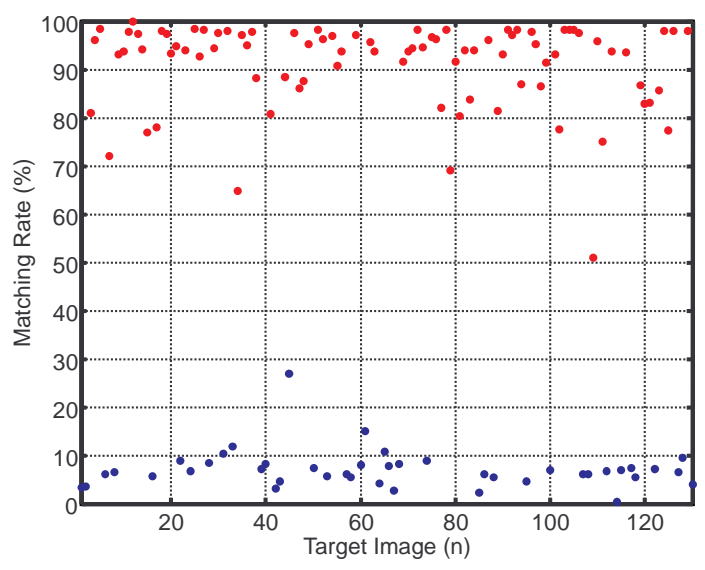

(c) using the GWHI method

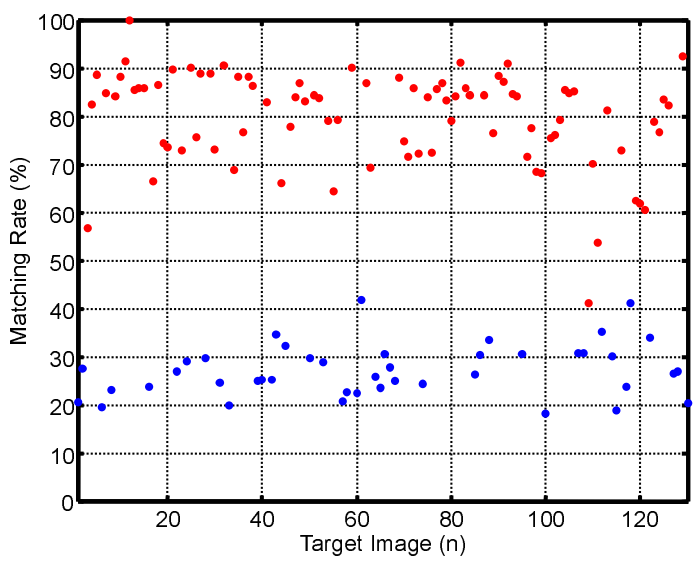

(e) using the CRG method

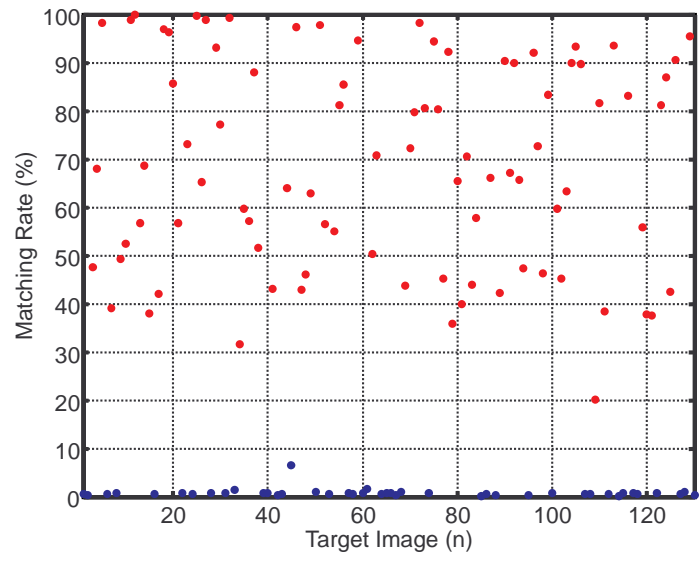

(b) using the MPHM method

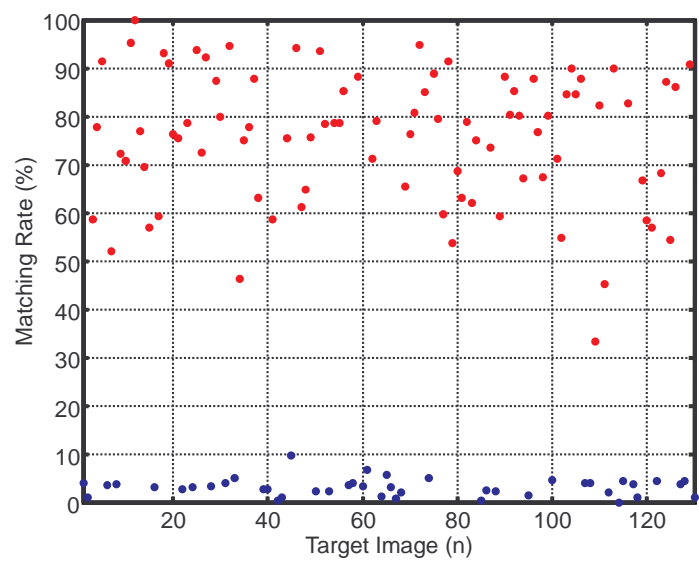

(d) using the refined GWHI

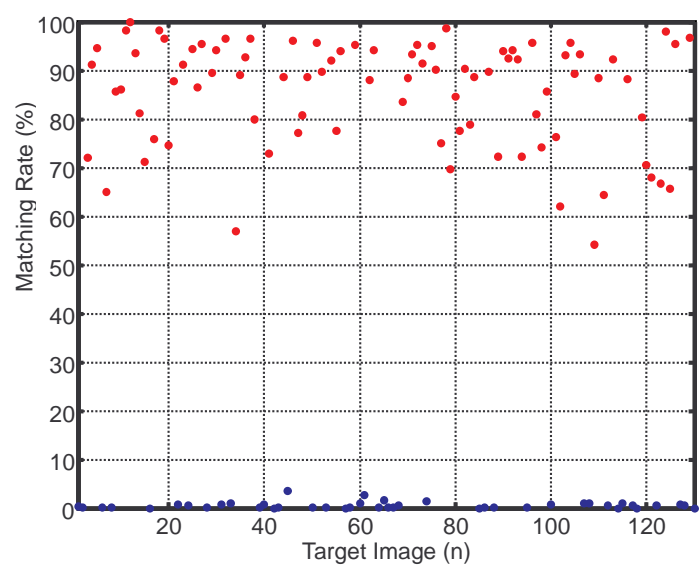

(f) using the $\mathrm{CECH}$ method

Figure 5. Histogram matching results obtained using the six histogram intersection methods. Matching data of yellow number plate images are marked as red dots, and matching data of white number plate images are marked as blue dots (darker if viewed in black-white picture). 
the cost of degenerated performance. As shown in Figure 5(d), the classification performance using the refined GWHI method is only slightly better than that of the MPHM method.

Using Gevers' CRG method, a more stable matching results are shown in Figure 5(e) for both same class of plates and different classes of plates. This reflects the advantages that the CRG method is less sensitive to colour variations. However, as found in the figure, the inter-class distance is also reduced. This is due to the fact that the CRG is computed in a very compact neighbourhood (each neighbouring point is only one pixel away from the central point). For compound colour objects, such as vehicle number plates, many areas appear as uniform in such small neighbourhood. This results in large amount of null colour ratio gradients in all channel, which dominates the CRG histogram matching results. As this result, CRG histograms cannot well reflect the colour difference between yellow plates and white plates.

Using Jia's CECH method, a performance comparatively good to that of the GWHI method can be obtained which is shown in Figure 5(f). Note that, the average processing time is only 22 milliseconds.

\section{Conclusions}

This paper introduces and compares six histogram-based image matching methods, i.e., the conventional histogram intersection (HI) method, Wong and Cheung's merged palette histogram matching (MPHM) method, Jia et al.'s Gaussian weighted histogram intersection (GWHI) method, the refined GWHI , Gevers' colour ratio gradient (CRG) method, and Jia et al.'s colour edge cooccurrence histogram $(\mathrm{CECH})$ method. These methods are tested on vehicle number plate images for number plate classification. Experimental results disclose that, among the investigated six methods, the CRG method is the fastest one. The matching performance of the GWHI method is the best one. When both speed and matching performance are concerned, the $\mathrm{CECH}$ is the best one.

\section{References}

[1] D. Crandall and J. Luo. Robust color object detection using spatial-color joint probability functions. Proceedings of the 2004 IEEE Computer Society Conference on Computer Vision and Pattern Recognition, 1:379-385, 2004.

[2] B. Funt and G. Finlayson. Color constant color indexing. IEEE Transactions on Pattern Analysis and Machine Intelligence, 17(5):522-529, 1995.

[3] T. Gevers and W. Smeulders. Color constant ratio gradients for image segmentation and similarity of texture objects. Proceedings of the 2001 IEEE Computer Society Conference on Computer Vision and Pattern Recognition, 1:1825,2001

[4] W. Jia, H. Zhang, X. He, and Q. Wu. Image matching using colour edge cooccurrence histograms. Proceedings of the 2006 IEEE International Conference on Systems, Man, and Cybernetics, page to appear, 2006.

[5] W. Jia, H. Zhang, X. He, and Q. Wu. Refined gaussian weighted histogram intersection and its application in number plate categorization. Proceedings of the 3rd International Conference on Computer Graphics, Imaging and Visualization, page to appear, 2006.

[6] W. Jia, H. Zhang, X. He, and Q. Wu. Symmetric colour ratio gradients in spiral architecture. Proceedings of the 18th International Conference on Pattern Recognition, page to appear, 2006.

[7] S. K. Nayar and R. M. Bolle. Reflectance ratio: A photometric invariant for object recognition. Proceedings of the 4th International Conference on Computer Vision, pages 280 285, 1993.

[8] M. J. Swain and D. H. Ballard. Indexing via color histograms. Proceedings of the 3rd International Conference on Computer Vision, pages 390-393, 1990.

[9] M. J. Swain and D. H. Ballard. Color indexing. International Journal of Computer Vision, 7(1):11-32, 1991.

[10] K.-M. Wong, C.-H. Cheung, and L.-M. Po. Merged-color histogram for color image retrieval. Proceedings of the 2002 International Conference on Image Processing, 3:949-952, 2002.

[11] X. Wu. Efficient statistical computations for optimal color quantization. Graphics Gems, 2:126-133, 1991. 